\title{
EDITORIAL
}

\section{Special issue for stem cells: a multi-perspective look at stem cells}

\author{
Qi ZHOU \\ State Laboratory of Reproductive Biology, Institute of Zoology, Beijing 100101, China
}

(C) Higher Education Press and Springer-Verlag Berlin Heidelberg 2010

The past few years may have been a golden time in the history of stem cell research. The unique properties stem cells possess, to proliferate indefinitely and to differentiate into multiple kinds of cells, make these cells wonderful platforms for studying organism development and valuable sources for regenerative medicine. Many research teams worldwide have joined this area and contributed at multiple levels. Nowadays, stem cell research has been an integration of multiple disciplines ranging from basic biological studies of genetic and epigenetic regulation of cell state, to translational biomedical studies on drug discovery and clinical treatment, and to biotechnology such as new biomaterials exploration. Taking the most exciting advances as an example, in 2006 Yamanaka and colleagues generated induced pluripotent stem (iPS) cells by ectopic expression of four transcription factors in somatic cells. iPS cells soon became a focus of mechanistic studies of epigenetic determination of cell fate, such as histone modification, DNA methylation and non-protein-coding RNAs. Meanwhile, disease-specific iPS cells are largely used for high-throughput drug discovery. And many teams have already performed iPS cell-based transplantation therapy in rodent models. Aiming at better utilizing iPS cells, new technologies such as single cell imaging and mRNA mediated gene transfection are being explored. Based on these advancements, we believe there will be more fascinating discoveries in stem cell research in the near future.

In this special issue, authors of eleven articles share with us their new findings and ideas on many different aspects of stem cell research. Among them, Cao et al. report that recombinant OCT4 and NANOG proteins have different effects on cultured human fibroblast cells, indicating different mechanisms in epigenetic reprogramming. Xiao et al. report their screening for self-renewal factors using a combination of mRNA and comparative genomic hybridization $(\mathrm{CGH})$ microarray assays of human embryonic stem cells. For differentiation and cell transplantation therapy, Yang et al. report that prostaglandin E2 can promote hematopoietic differentiation from human embryonic stem cells. Li et al. discuss the features of two different neural progenitors existing in the brain and their therapeutic potential for spinal cord injuries. Sun et al. review the role of bone morphogenetic protein (BMP) signaling in regulation of neural stem cells during neural lineage development. Liu et al. review the stem cells in bone biology and their potential clinical applications for bone regeneration through collaborations among bone biologists, stem cell biologists and biomaterial scientists. Huang et al. review the contradictions of cancer stem cell theory and discuss the advancement of cancer therapy by targeting cancer stem cells. From a biotechnical perspective, Li et al. report their versatile methods for lineage tracing during embryonic stem cell differentiation by utilizing specific promoterdriven GFP expression. Yang et al. review the technical advancement of iPS cells and their potential applications in disease modeling, high-throughput drug screening and toxicological testing. Beyond mammalian stem cells, Su et al. report their study to analyze the patterns of stem cell differentiation in the processes of in vitro somatic embryogenesis in Arabidopsis; and Liu et al. review the distinct features of stem cells and their roles in shoot apical meristems. We hope these articles demonstrate some of the fascinating aspects of stem cell biology to all of our readers.

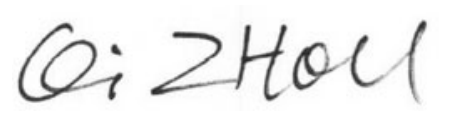

Qi ZHOU

Associate Editor-in-Chief

Frontiers in Biology 